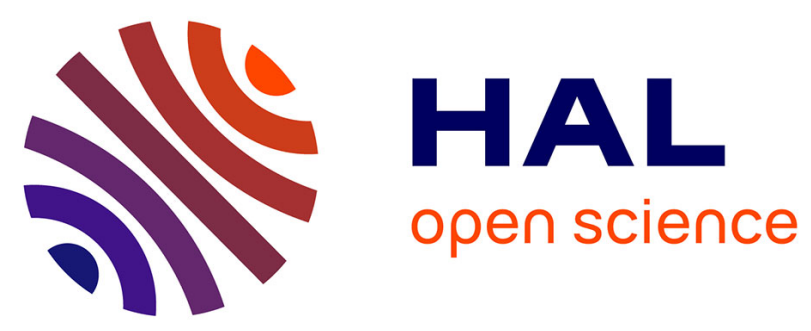

\title{
Real-Time Assessment of Ultrasound-Mediated Drug Delivery Using Fibered Confocal Fluorescence Microscopy
}

Marc Derieppe, Anna Yudina, Matthieu Lepetit-Coiffé, Baudouin Denis de Senneville, Clemens Bos, Chrit Moonen

\section{To cite this version:}

Marc Derieppe, Anna Yudina, Matthieu Lepetit-Coiffé, Baudouin Denis de Senneville, Clemens Bos, et al.. Real-Time Assessment of Ultrasound-Mediated Drug Delivery Using Fibered Confocal Fluorescence Microscopy. Molecular Imaging and Biology, 2013, 15 (1), pp.3-11. hal-00994203

\section{HAL Id: hal-00994203 \\ https://hal.science/hal-00994203}

Submitted on 28 Dec 2017

HAL is a multi-disciplinary open access archive for the deposit and dissemination of scientific research documents, whether they are published or not. The documents may come from teaching and research institutions in France or abroad, or from public or private research centers.
L'archive ouverte pluridisciplinaire HAL, est destinée au dépôt et à la diffusion de documents scientifiques de niveau recherche, publiés ou non, émanant des établissements d'enseignement et de recherche français ou étrangers, des laboratoires publics ou privés. 


\title{
Real-Time Assessment of Ultrasound-Mediated Drug Delivery Using Fibered Confocal Fluorescence Microscopy
}

\author{
Marc Derieppe, ${ }^{1,2}$ Anna Yudina, ${ }^{1,2}$ Matthieu Lepetit-Coiffé, ${ }^{1}$ \\ Baudouin Denis de Senneville, ${ }^{1,3}$ Clemens Bos, ${ }^{2}$ Chrit Moonen ${ }^{1,2}$ \\ ${ }^{1}$ Laboratory for Molecular and Functional Imaging: From Physiology to Therapy, FRE 3313-CNRS and University Bordeaux Segalen, 146, \\ rue Léo Saignat, Case 117, 33076, Bordeaux cedex, France \\ ${ }^{2}$ Imaging Division, University Medical Center Utrecht, Heidelberglaan 100P.O. Box 85500,3508 GA, Utrecht, Netherlands \\ ${ }^{3}$ Institut de Mathématiques de Bordeaux, UMR 5251-CNRS-Université Bordeaux 1-INRIA, Bordeaux, France
}

\begin{abstract}
Purpose: Transport across the plasma membrane is a critical step of drug delivery for weakly permeable compounds with intracellular mode of action. The purpose of this study is to demonstrate real-time monitoring of ultrasound (US)-mediated cell-impermeable model drug uptake with fibered confocal fluorescence microscopy (FCFM).

Procedures: An in vitro setup was designed to combine a mono-element US transducer, a cell chamber with a monolayer of tumor cells together with SonoVue microbubbles, and a FCFM system. The cell-impermeable intercalating dye, SYTOX Green, was used to monitor US-mediated uptake. Results: The majority of the cell population showed fluorescence signal enhancement $10 \mathrm{~s}$ after US onset. The mean rate constant $k$ of signal enhancement was calculated to be $0.23 \pm 0.04 \mathrm{~min}^{-1}$.

Conclusions: Feasibility of real-time monitoring of US-mediated intracellular delivery by FCFM has been demonstrated. The method allowed quantitative assessment of model drug uptake, holding great promise for further local drug delivery studies.
\end{abstract}

Key Words: Drug delivery, Biological barrier, Plasma membrane permeabilization, Ultrasound bioeffects, Fibered confocal fluorescence microscopy, Pharmacokinetic parameters, SYTOX Green, US-mediated drug delivery

\section{Introduction}

$\mathrm{D}$ elivery to the target site at high concentration, while minimizing accumulation in healthy organs, is a crucial factor in determining the efficacy of a drug [1]. It is of a particular interest when treatment involves compounds with

Electronic supplementary material The online version of this article (doi:10.1007/s11307-012-0568-9) contains supplementary material, which is available to authorized users.

Correspondence to: Chrit Moonen; e-mail: c.moonen@umcutrecht.nl toxic side effects, for example chemotherapy agents [2]. Efficient delivery requires the therapeutic agent to cross biological barriers such as the endothelium for intravenously injected molecules, gastrointestinal barriers for orally administered drugs, or the plasma membrane for drugs that need to reach the intracellular compartment in order to exert their action. The permeability of biological barriers to a drug is influenced by the physico-chemical properties of the drug such as molecular weight, solubility, lipophilicity, and ionization [3].

It has been demonstrated in vitro [4-6] and in vivo $[4,6]$ that ultrasound (US) can enhance both vascular and plasma membrane permeability, thus locally facilitating compound delivery. However, the recent literature indicates that the 
debate about underlying mechanisms of plasma membrane sonoporation is still ongoing [7-9]. In vitro imaging techniques such as scanning electron microscopy $[10,11]$ and fluorescence microscopy [12] play an increasingly important role in the investigation of different aspects of US-mediated drug delivery. In vivo imaging modalities, such as magnetic resonance imaging [13] and optical imaging [14, 15], are also being applied to US-mediated drug delivery monitoring and evaluation. Although these techniques allow the observation of US-induced bioeffects, currently they are not able to provide real-time follow-up of sonoporation and kinetic assessment on the cellular level.

On the other hand, light microscopy of US-mediated intracellular drug delivery has been achieved with the highspeed Brandaris camera [16] that allows real-time imaging of events on nanosecond scale, such as the interaction between cells and microbubbles subjected to ultrasound [17, 18]. However, the short time range of acquisition, high cost, as well as difficulties with adaptation to in vivo use and fluorescence imaging limit the potential applications of this technique.

Fibered confocal fluorescence microscopy (FCFM) was designed for real-time in situ and in vivo cellular imaging in small animals and humans [19-21]. With up to eight frames per second and small size, this device could also be well suited for studying dynamic processes involved in the local drug delivery, such as real-time monitoring of the drug uptake by cells and assessing its kinetic parameters. In this context, recent studies $[15,22]$ have shown the use of cellimpermeable intercalating dyes as smart probes to demonstrate US-induced membrane permeabilization with epifluorescence microscopy; these chromophores (for example, SYTOX Green, TO-PRO-3) exhibit 100-1,000-fold fluorescence signal enhancement upon binding to nucleic acids. Due to the novelty of the approach and lack of the quantification assessment with FCFM or other modalities, we have first chosen to work with an in vitro model.

In this paper, we demonstrate the feasibility of monitoring of the US-mediated live cell uptake of a "smart probe" (model drug) with FCFM in vitro. For this purpose, a setup allowing FCFM real-time acquisitions during US exposure was designed, consisting of the combination of a monoelement transducer, an optical imaging and ultrasound compatible cell chamber, and a FCFM system. The resulting SYTOX Green fluorescence enhancement entailed by DNA intercalation in the cell nuclei was assessed and fitted to a two-compartment model, to study uptake kinetics.

\section{Materials and Methods}

An in vitro setup was built to allow US-mediated fluorophore uptake to be monitored by real-time fluorescence imaging. Figure 1a shows the location of the different elements placed in a $37{ }^{\circ} \mathrm{C}$ water bath. A cell culture chamber was positioned horizontally so that the adherent cell monolayer was attached to the upper membrane, which made contact with the FCFM Z microprobe. For all studies, the mono-element US transducer was placed below the culture chamber at a distance of $8 \mathrm{~mm}$ from the cell monolayer [22]. The water bath served as a coupling medium for US propagation.

\section{Ultrasound Setup}

A 5.8-mm-diameter mono-element (Imasonic, Lyon, France) US transducer was used. US protocol was the same as the one used in previous studies $[22,23]$, which was shown to result in a maximum delivery area while minimizing cell detachment: main frequency $=1.5 \mathrm{MHz}$, pulse repetition frequency $=1 \mathrm{kHz}$, duty cycle $=20 \%$, electrical effective power $=1.0 \mathrm{~W}$, US triggering $=10 \mathrm{~s}$ (Fig. 1b), and exposure time $=30 \mathrm{~s}$. Measured acoustical pressure was maximal ( $0.88 \mathrm{MPa}$ peak-to-peak) at $8 \mathrm{~mm}$ distance. This set of ultrasound parameters only generated stable cavitation.

\section{Fluorophores and Microbubbles}

Cell-impermeable dye SYTOX Green (Invitrogen Life Technologies, Saint-Aubin, France) (excitation/emission $=504 / 523 \mathrm{~nm}$ ) was used in this study. This fluorophore is a small-molecule (molecular weight $600 \mathrm{Da}$ ), high-affinity, intercalating agent that is virtually nonfluorescent in aqueous solution and exhibits a 100- to 1,000-fold increase in fluorescence upon binding to nucleic acids. It is cellimpermeable under normal physiological conditions but easily penetrates compromised plasma membranes. The final concentration used in the medium was $2 \mu \mathrm{M}$. A commercially available ultrasound contrast agent (Sonovue, Bracco, Milan, Italy) served for the creation of the cavitation effect. The microbubbles contain sulfur hexafluoride and are delivered as a lyophilized phospholipids/poly(ethylene glycol)/palmitic acid powder, stored under $\mathrm{SF}_{6}$ gas. Upon addition of a saline solution, a suspension of microbubbles $\left(2 \times 10^{8}\right.$ microbubbles/ $\mathrm{ml}$, mean diameter $2.5 \mu \mathrm{m}$, range 0.7 to $10 \mu \mathrm{m}$ ) stabilized by a lipidic monolayer is produced [24]. During sonication experiments, the final concentration of microbubbles in the culture chamber was $3 \times 10^{7}$ microbubbles $/ \mathrm{ml}$.

\section{Cell Culture}

ATCC C6 rat glioma cells were seeded into OptiCell ${ }^{\mathrm{TM}}$ culture chambers (Thermo Fischer Scientific, Rochester, NY) supplemented with $10 \mathrm{ml}$ of complete medium as described previously [22]. The culture chamber has two parallel membranes of $50 \mathrm{~cm}^{2}$ attached to a rectangular frame with two access ports. The membranes of the culture chamber are $75 \mu \mathrm{m}$ thick, $2 \mathrm{~mm}$ apart, and allow free gas exchange and optical imaging. The membranes show neither energy loss nor standing waves during US application [23].

\section{Fibered Confocal Fluorescence Microscopy}

For real-time fluorescence imaging, a fibered confocal fluorescence microscopy system was used (CellVizio Lab, Mauna Kea Technologies, Paris, France). The FCFM system with a 488-nm laser excitation wavelength was well-adapted to SYTOX Green fluorophore excitation, spectral emission was filtered (505 nm to $700 \mathrm{~nm}$ ), and images were acquired at an $8.5-\mathrm{Hz}$ frame rate. 
a
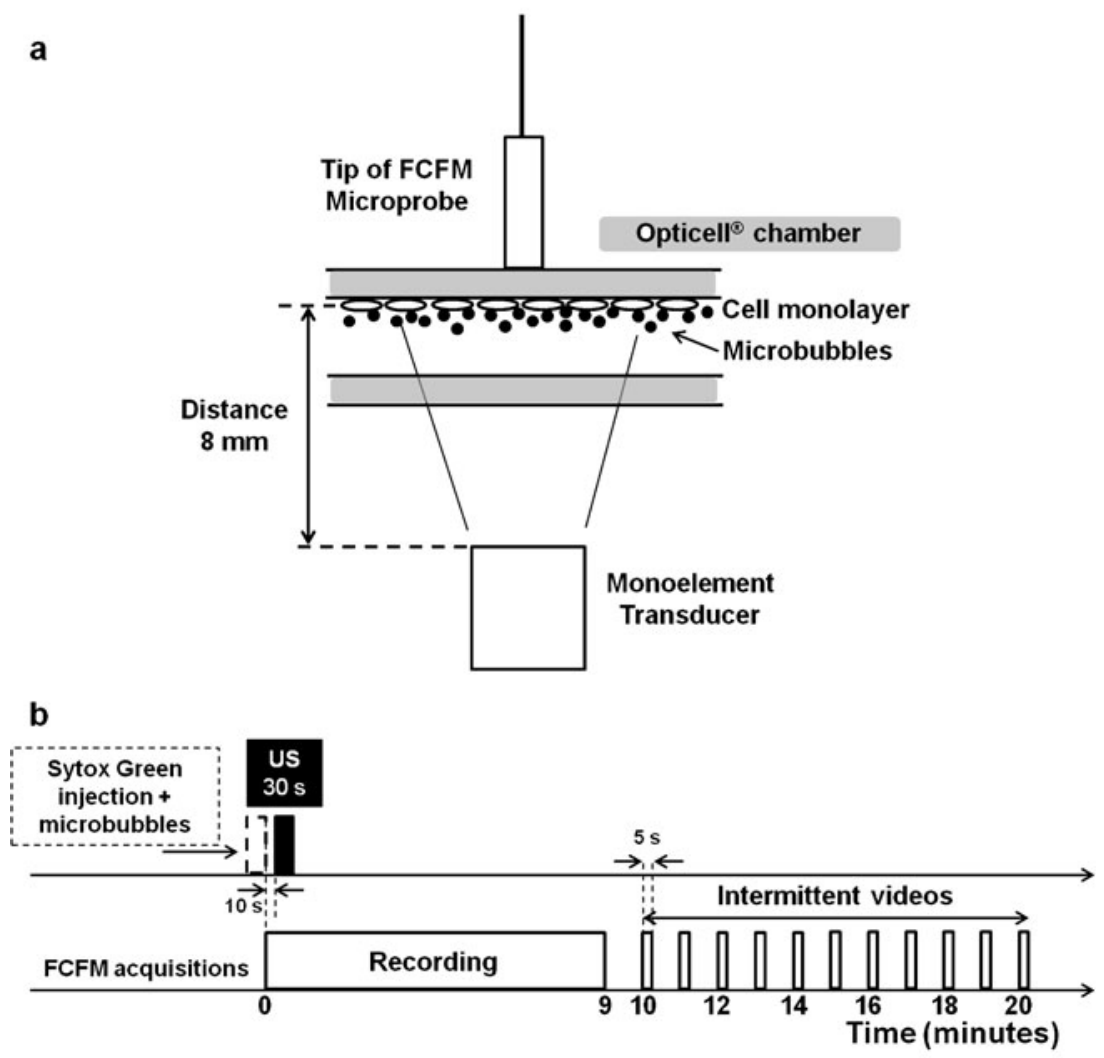

Fig. 1. a In vitro setup for ultrasound (US)-mediated cell uptake monitoring by fibered confocal fluorescence microscopy (FCFM). The cell monolayer was positioned horizontally so that the adherent cell monolayer was attached to the upper membrane, which was in direct contact with the FCFM Z microprobe. For all studies, the mono-element US transducer was placed below the culture chamber at a distance from the cell monolayer equal to the maximal measured acoustical pressure (8 $\mathrm{mm}$ ) of the transducer. $\mathbf{b}$ Time line of the experiments. SYTOX Green and microbubbles were injected immediately before the imaging. The first video was acquired continuously during $9 \mathrm{~min}$ and started $10 \mathrm{~s}$ before US exposure. US exposure lasted $30 \mathrm{~s}$. After the first 9-min video, 5-s videos were acquired every minute to limit photobleaching effect.

The FCFM device consists of a laser-based optoelectronics unit, a Z microprobe (Z1800, Mauna Kea Technologies, Paris, France) composed of a bundle of 12,000 optical fibers that is the link between the laser scanning unit and a distal micro-objective. The two-dimensional bundle is scanned by the laser with a $4-\mathrm{kHz}$ oscillating mirror and a galvanometric mirror. Each fiber has an illumination-emission cycle, and the same path is followed for excitation light and fluorescence emission; the latter is diverted by a dichroic filter to a photodetector. The $\mathrm{Z}$ probe used has a $100-\mu \mathrm{m}$ working distance, which allows placing the optical section beyond the $75-\mu \mathrm{m}$-thick culture chamber membrane, at the cell monolayer. The $\mathrm{Z}$ probe provides $70 \mu \mathrm{m}$ axial resolution, $3.9 \mu \mathrm{m}$ lateral resolution, and $600 \times 500 \mu \mathrm{m}$ field of view, allowing observation of a large number of cells, and, thus, the computation of statistics over a cell population.

\section{FCFM System Linearity}

Signal linearity of the FCFM system was studied in a dilution series of SYTOX Green in salmon sperm DNA solution (dilution $1 \mathrm{mg}$ / ml, Invitrogen Life Technologies, Saint-Aubin, France): 11 Eppendorf tubes containing SYTOX Green concentrations of 0 to
$70 \mu \mathrm{M}$ were obtained by serial dilution. Then, for each concentration, the tip of the FCFM microprobe was immersed for $5 \mathrm{~s}$ to measure fluorescence intensity. The system response was considered linear when Pearson's correlation coefficient $r^{2}$ was greater than 0.99 .

\section{Live Cell Microscopic Imaging}

To compare nuclei detection with FCFM to epifluorescence microscopy, live cell imaging was performed using an epifluorescence microscope (Leica DMR, Leica Microsystems, Wetzlar, Germany) equipped with a $\times 10$ objective (HC PL Fluotar $10 \mathrm{X}$ dry $0.3 \mathrm{NA}$ ) and a set of filters appropriate for SYTOX Green. Images were acquired with a CoolSnapHQ camera (Roper Scientific, Evry, France), using a 5-ms exposure time.

\section{Experimental Protocol}

The regions with US-exposed cells were identified by drawing circles on the membrane of the culture chamber [22]. The tip of the FCFM microprobe was placed in the center in line with the 
ultrasound transducer (Fig. 1a). Fresh complete medium containing $3 \times 10^{7}$ perfluorocarbon microbubbles together with SYTOX Green dye was injected into the culture chamber prior to experiments. The US transducer, the culture chamber, and the tip of the FCFM microprobe were then immersed in a water bath at $37{ }^{\circ} \mathrm{C}$ (nondegassed water).

FCFM imaging lasted for $20 \mathrm{~min}$ in total. The first video was acquired during $9 \mathrm{~min}$ without interruptions and started $10 \mathrm{~s}$ before the sonication to observe signal enhancement due to US-mediated permeabilization (Fig. 1b). Cells were exposed to US for $30 \mathrm{~s}$. After the first 9-min video, 5-s videos were acquired every minute to limit the photobleaching effect. The experiment was repeated three times, independently, using the same exposure and data acquisition protocol on a different region in the cell culture chamber.

\section{Data Analysis}

Data post-processing was done with ImageJ software (NIH, Bethesda, MD, USA) using standard routines, and custom scripts written in IDL 8.0 (Exelis Visual Information Solutions). First, the proprietary .mkt files (Mauna Kea Technologies) were converted to TIFF images. Then, the individual videos were combined for analysis of the whole imaging session. Analyses were performed after resampling at 1 -s intervals, from the $8.5-\mathrm{Hz}$ acquisition frame rate set by default by the system software. In order to remove the background signal corresponding to fiber bundle auto-fluorescence, a pixel-by-pixel baseline correction was performed on the basis of the five first resampled images in the first acquisition. The final image of the imaging session served as a reference to delineate cell nuclei that were clearly noticeable; a minimum intensity threshold was manually performed with ImageJ software for this delineation. This delineation resulted in the labeling of disjoint, independent regions, one per cell. Then, for analyses pertaining to the population of cells in an experiment, these regional maxima were averaged to reflect the enhancement behavior of the whole sample.

For analysis of the fluorescence enhancement over time, a twocompartment model was used consisting of an extracellular space and the cellular compartment, separated by a plasma membrane. Then, the signal intensity $(I)$ depends on time as:

$$
I(t)=A\left[1-e^{-k(t-T)}\right]
$$

Here $A$ is the asymptotic signal enhancement, $T$ the time of fluorescence onset, and $k$ the rate constant. These three parameters were obtained from a Levenberg-Marquardt least-squares fit to the first 9-min temporal evolution of signal maximum in each region. This fit was restricted to the first 9-min video to limit the influence of photobleaching. The signal maximum was chosen because it is robust to errors in determination of the cell outlines and to cell motion.

For each experiment, the number of enhanced nuclei over time was counted. To this end, for each enhancing region, the mean and standard deviation (SD) from 0 to $8 \mathrm{~s}$ were calculated, based on the signal maximum in the region. When the signal maximum exceeded the baseline by $5 \mathrm{SD}$, the cell was considered as permeabilized. Total number of permeabilized cells and the time point of first enhancement were recorded.

The goodness of fit of the model for describing the signal evolution was estimated by means of squared Pearson's correlation coefficient $\left(r^{2}\right)$. When $r^{2}>0.95$, the model was considered sufficient to accurately describe the data. Values are reported as mean \pm standard deviation.

\section{Results}

A novel in vitro setup was designed to study kinetics of model drug uptake by live cells before, during, and after sonoporation in continuous mode.

\section{System Linearity}

The linearity of the system was assessed with serial dilutions of SYTOX Green in Eppendorf tubes at three laser powers (Fig. 2). At $2.86 \mathrm{~mW}$, a clear linearity was observed (fit of the 7 points from 0 to $13.8 \mu \mathrm{M}$, yielding $\left.r^{2}=0.992\right)$ until the signal was clipped by the analog-to-digital conversion (i.e., $>8,191$ intensity arbitrary units) at $21 \mu \mathrm{M}$, so that only a narrow concentration range could be exploited due to signal clipping. We also observed linearity at the two other laser powers, for concentrations up to 31 and $47 \mu \mathrm{M}$ at 1.22 and $0.82 \mathrm{~mW}$, respectively. Thus, we chose a $0.82-\mathrm{mW}$ laser power so that, in the uptake experiments, the signal intensity depended linearly on concentration and intensities were in the dynamic range of the detector, even for the highest concentrations expected.

\section{Comparison of Epifluorescence Microscopy and Fibered Confocal Fluorescence Microscopy}

Images acquired with epifluorescence microscopy using $\mathrm{a} \times 10$ magnification and FCFM are shown in Fig. 3. Comparison of the two snapshots (Fig. 3b, e) and their zoomed in (Fig. 3c, f) showed that FCFM spatial resolution was sufficient to clearly distinguish individual cell nuclei similar to an epifluorescence microscope with $\mathrm{a} \times 10$ objective.

In all cases, as long as US exposure was not triggered, no activation of the signal could be observed (left column), apart from occasional dead cells whose membrane is damaged (Fig. 3a, d). Similarly, no signal intensity enhancement was noticeable in the absence of microbubbles with cells subjected to ultrasound. Conversely, the combination of US exposure and microbubbles resulted in a characteristic nuclear staining registered with both techniques (Fig. 3b, e), demonstrating US-mediated SYTOX Green uptake.

\section{Real-Time Imaging of Model Drug Uptake}

The real-time US-mediated SYTOX Green delivery was registered with FCFM (Fig. 4). In accordance with the observations mentioned above, signal intensity enhancement onset was not observable before US application, apart from occasional dead cells whose signal was stable (Fig. 4a). US application at $10 \mathrm{~s}$ of imaging was followed by the appearance of circular areas of increasing fluorescence signal intensity, corresponding to cell nuclei. The diameter of 


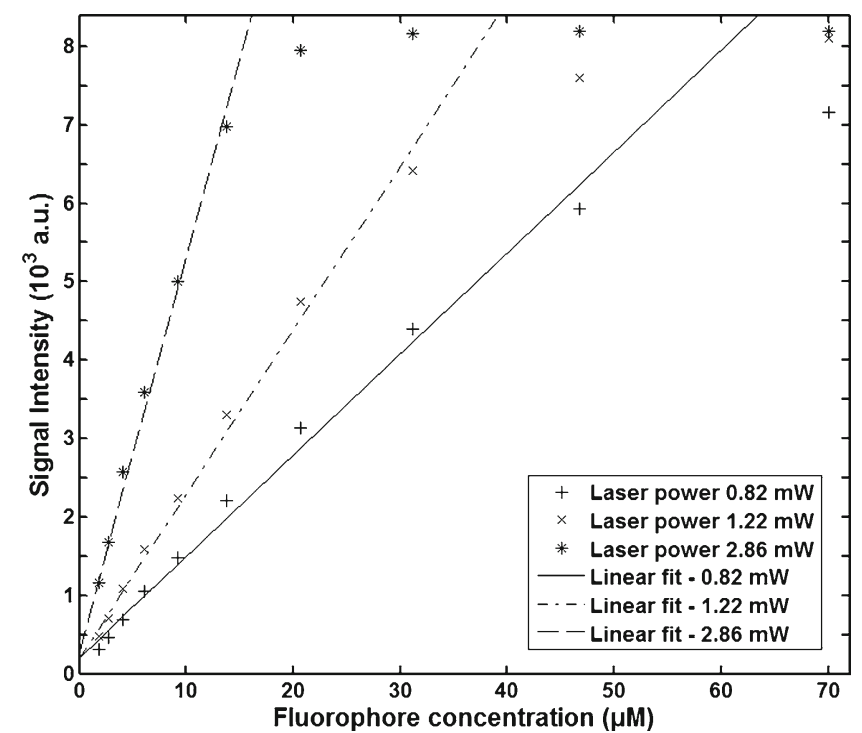

Fig. 2. Fluorescence signal intensity as a function of fluorophore concentration at three laser powers, and the corresponding linear fits: $0.82 \mathrm{~mW}$ (crosses and solid line), $1.22 \mathrm{~mW}$ (diagonal crosses and dashed dotted line), and $2.86 \mathrm{~mW}$ (asterisks and dashed line). Fluorophore concentrations up to 47,31 , and $14 \mu \mathrm{M}$ were considered for the linear fits for the $0.82,1.22$, and $2.86 \mathrm{~mW}$ laser powers, respectively. The $0.82-\mathrm{mW}$ laser power was chosen so that the signal intensity used in the uptake experiments, in the expected concentration range, was linear without saturation.

circular areas increased during the first seconds of signal intensity enhancement, stabilizing at 5-10 $\mu \mathrm{m}$ (see Online supplementary video).

The evolution of the number of enhanced nuclei over the first 2 min of acquisition in the three independent experiments is reported in Fig. 5. No nuclear fluorescence enhancement could be noticed in any of the control experiments, lacking US exposure or microbubbles (Fig. 5a and b). The number of fluorescent nuclei increased rapidly until reaching the total number of permeabilized cells $1 \mathrm{~min}$ and $50 \mathrm{~s}$ after US onset (Fig. 5a). In the early stage of cell uptake activation, a correlation is noticeable between the onset of US and the time when the first cells in the field of view show signal activation, as defined by the mean baseline $+5 \mathrm{SD}$ criterion (Fig. $5 \mathrm{~b}$ ). In addition, the majority of permeabilized cells reach this threshold between 2.0 and $3.0 \mathrm{~s}$ after US onset (Fig. $5 \mathrm{c}-\mathrm{e}$ ): the distributions 1 (Fig. 5c), 2 (Fig. 5d), and 3 (Fig. 5e) indicated a median of $2.0 \mathrm{~s}(1.0-5.5 \mathrm{~s}), 2.5 \mathrm{~s}(1.0-8.0 \mathrm{~s})$, and $3.0 \mathrm{~s}(1.0-7.0 \mathrm{~s})$, respectively. Lower and upper quartile values are given in brackets.

\section{Determination of the Rate Constant $k$ and Accuracy of the Two-Compartment Model}

The temporal evolution of the signal maximum in two representative cell nuclei, as well as that of the mean signal intensity of the maxima, is reported in Fig. 6a and c, respectively. The Levenberg-Marquardt least-squares method was applied to fit a two-compartment model, resulting in an exponential pattern (Eq. 1). To limit the influence of photobleaching effect, we chose to fit only the first 9-min acquisition. With regard to the individual cell nuclei (Fig. 6a), $0.15 \mathrm{~min}^{-1}$ $\left(r^{2}=0.998\right)$ and $0.18 \mathrm{~min}^{-1}\left(r^{2}=0.998\right)$ time constants were found. In addition, three independent experiments allowed the labeling of 553 regions in total. Of these cells, $87 \%$ (483 out of 553 regions) showed enhancement behavior consistent with the two-compartment model $\left(r^{2}>0.95\right)$. As a population (Fig. $6 \mathrm{c}$ ), these cells showed a characteristic mean rate constant $k$ for SYTOX Green uptake of $0.23 \pm 0.04 \mathrm{~min}^{-1}(n=3)$.

Moreover, a clear correlation between US onset and early stage of fluorescence signal enhancement in these two cell
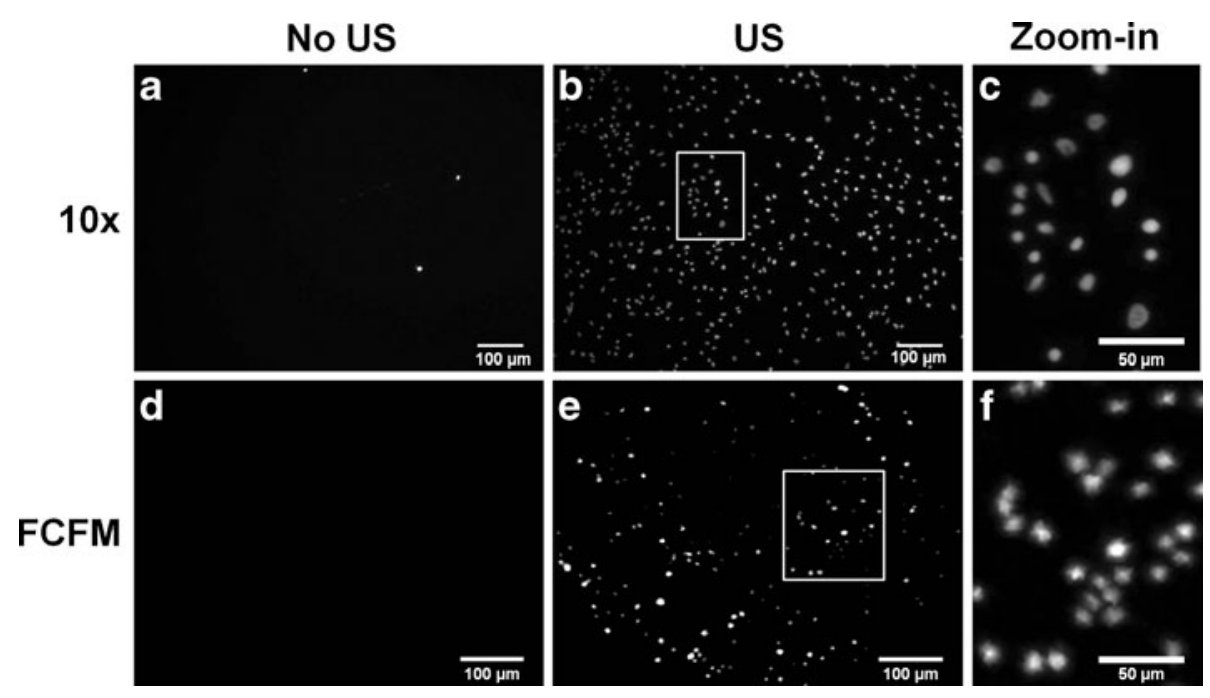

Fig. 3. Comparison between epifluorescence microscopy at $\times 10(a, b$, and $c)$ and FCFM images $(d$, e, and $f)$. For each of the two magnifications, the left column shows images acquired without US, the middle column shows resulting images with the cell monolayer subjected to ultrasound (bar=100 $\mu \mathrm{m}$ ). The right column displays the zoomed in images in the middle column (white rectangles) (bar $=50 \mu \mathrm{m})$. FCFM spatial resolution was sufficient to clearly distinguish individual cell nuclei. 

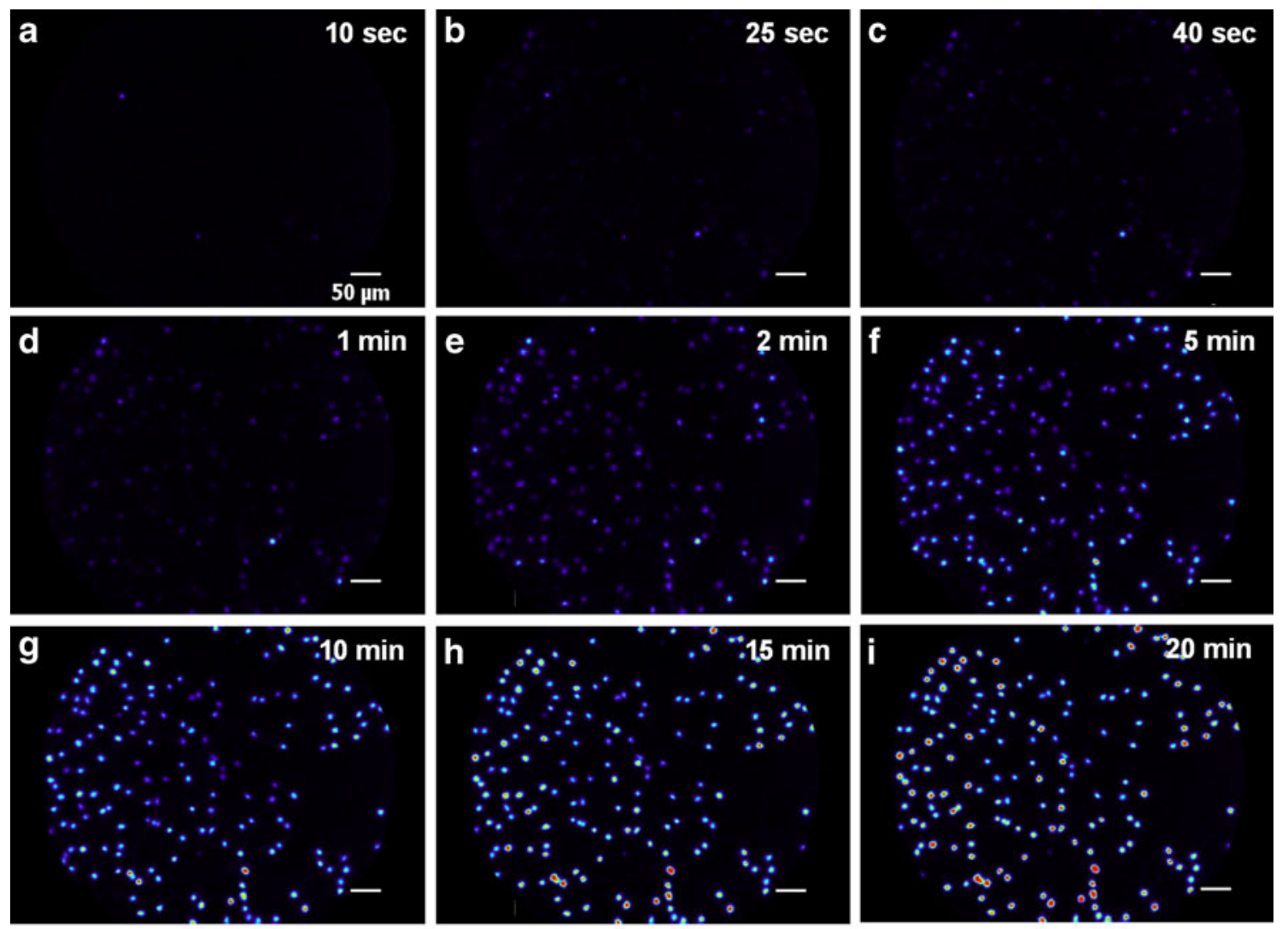

0

4500

8191

Fig. 4. Snapshots of a representative 20-min imaging session. Images acquired at the onset of US exposure (10 s) (a), during (25 and $40 \mathrm{~s}$ ) (b, c), and after US exposure (from 2 to $20 \mathrm{~min})(\mathbf{d}-\mathbf{i})$. Bar $=50 \mu \mathrm{m}$.

nuclei (Fig. 6b), as well as for the mean signal enhancement (Fig. 6d), was noticeable, suggesting that most of the cells in the sample are permeabilized as soon as the US is triggered. The signal maximum, as well as the mean signal enhancement, continued to increase after US exposure and suggested that the membranes remain permeable at least at the scale of minutes assessed during the current experiment.

\section{Discussion}

The study of local drug delivery would benefit from in vitro and in vivo real-time monitoring of cellular uptake processes. Here, it was demonstrated that fibered confocal fluorescence microscopy can be used in an in vitro setup to monitor model drug uptake by cells subjected to ultrasound and microbubbles. US-mediated plasma membrane permeabilization was investigated in $\mathrm{C} 6$ rat glioma cells, using a combination of an ultrasound and FCFM compatible cell culture chamber, a mono-element US transducer, and a FCFM system. Cell-impermeable intercalating dye SYTOX Green was employed as a smart probe to study US-induced membrane permeabilization [15] and drug model uptake. Resulting kinetic parameters of the uptake could be assessed using this approach.
The individual images obtained with the FCFM have a field of view that is comparable to that of a $\times 10$ magnification objective of epifluorescence microscopy. Although, the image resolution is inferior to that of conventional microscopy, individual nuclei can be clearly observed, in agreement with previous studies [25], which is a big asset for studies of intracellular drug delivery.

The attractiveness of our FCFM setup is that it allows continuous recording before, during, and after US application, as opposed to conventional microscopy $[22,26]$. Based on a fiber optic imaging bundle, the FCFM microprobe design lacks geometrical constraints, which makes this approach suitable for translation to in vivo experiments that require setups with access limited by other instrumentation. In addition, the minimally invasive nature of FCFM makes it an attractive alternative to the dorsal skinfold window chamber [27, 28] that is often exploited in the context of local drug delivery [14].

With FCFM, the ability to observe single nuclei in a rather large field of view allows to identify and label them in the images and monitor the evolution of the fluorescence signal intensity at the individual cell level. Here, the temporal evolution of the signal intensity was fitted to a two-compartment model, allowing the assessment of kinetic parameters for each nucleus; in particular, the rate constant $k$ and the saturation signal $A$. 

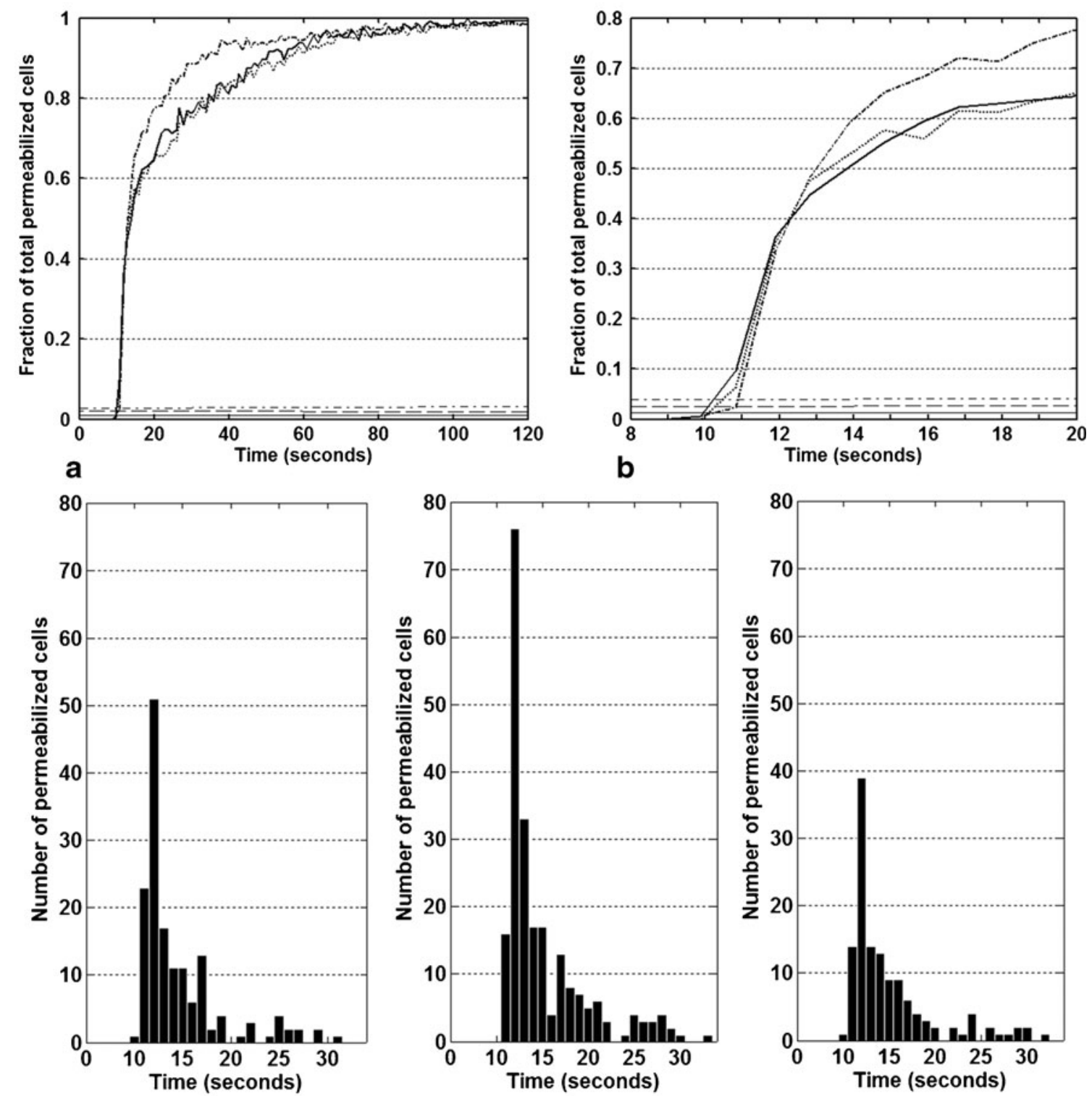

Fig. 5. a The fraction of total permeabilized cells as a function of the acquisition time, which is defined as the current number of permeabilized cells divided by the total number of permeabilized cells at the end of observation. US together with microbubbles in three separate experiments (bold solid line, bold dotted line, and bold dashed dotted line) and associated controls: neither US application nor presence of microbubbles (dashed line), US without microbubbles (solid line), and microbubbles without US application (dashed dotted line). $\mathbf{b}$ Twenty-second early stage of the fraction of permeabilized cells subjected to US (zoom in of the graph presented in a). c-e Corresponding histograms of distribution of the cell permeabilization. For each experiment, the fraction of permeabilized cells is plotted for 1 -s intervals.

The time of fluorescence onset was determined when the fluorescence signal was greater than $5 \mathrm{SD}$ of the mean baseline (from 0 to the first $8 \mathrm{~s}$ of imaging). While this criterion confounds with the activation time of the cells, the experiments give insight in the time scale of the internalization process. Most of the cells that took up the model drug showed evidence of SYTOX Green uptake immediately after US triggering, with a signal activation between 2 and $3 \mathrm{~s}$ after US onset, with a tail of up to a few seconds, indicating that the permeabilization and the subsequent uptake occur as soon as US exposure was triggered. A mean rate constant $k$ of $0.23 \pm 0.04 \mathrm{~min}^{-1}$ was found, which puts the time scale of the enhancement at several minutes. Previous studies [11, 17] found a resealing time scale significantly shorter than our current results; these contradictory results could be related to different US regimes used in these experiments or other yet unknown causes.

In this paper, a two-compartment model was chosen to describe our system, i.e., an extracellular space and the cellular compartment separated by a plasma membrane. For $87 \%$ of the individual cells, the uptake curves were consistent with a two-compartment model, i.e., $r^{2}>0.95$. 
a

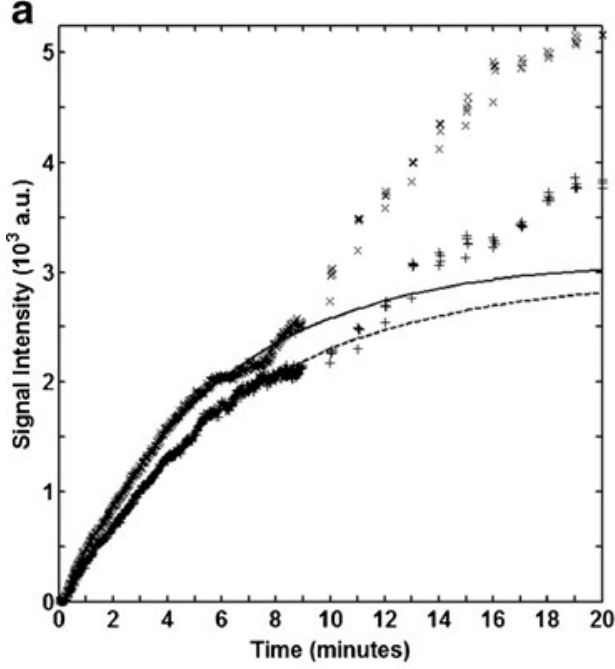

C

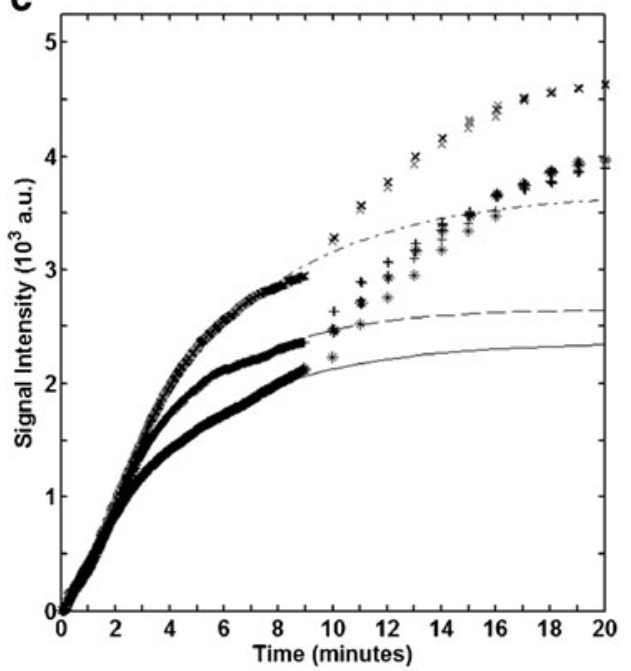

b

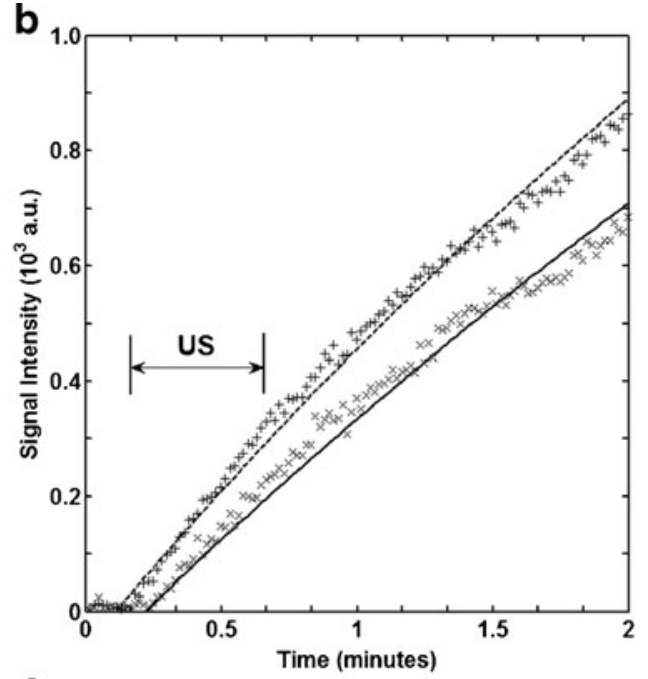

d

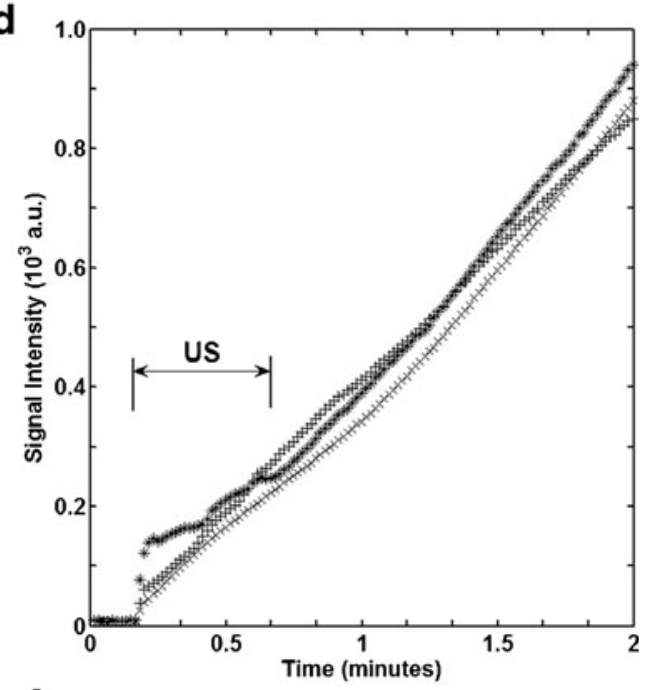

Fig. 6. a Maximum signal intensity of two cell nuclei as a function of time. The 9-min fit yielded: $k=0.15 \min ^{-1}\left(r^{2}=0.998\right.$, dashed line), $k=0.18 \mathrm{~min}^{-1}\left(r^{2}=0.998\right.$, solid line). $\mathbf{b}$ Early phase of the signal enhancement (zoom in of the first $2 \mathrm{~min}$ in the graph in a). The correlation between the US application and the onset of signal intensity in each of these two individual cells is noticeable. c Fluorescence signal intensity enhancement of the population of cells (mean of the maxima of cell nuclei) as a function of time. Rate constant determined by the 9-min fit: $k=0.23 \mathrm{~min}^{-1}$ (solid line), $0.27 \mathrm{~min}^{-1}$ (dashed line), and $0.20 \mathrm{~min}^{-1}$ (dashed dotted line), entailing a mean rate constant of $0.23 \pm 0.04 \mathrm{~min}^{-1}$. $\mathrm{d}$ Early phase of signal enhancement (first 2 min of the graph in c). The correlation between the US application and the onset of signal intensity is also noticeable.

Nevertheless, $13 \%$ of the regions in the field of view did not fit with this two-compartment model; this could partially be explained by a photobleaching effect that limits the fluorescence intensity towards the end of the first 9-min acquisition (Fig. 6a). Since it is a measurement bias, photobleaching will decrease the $r^{2}$ value or may confound the measurement of the time constant $k$. Alternatively, these cells could have a truly different kinetic pattern which will be the subject of further investigation.

Moreover, instead of assessing the transport coefficients of the cell membrane exclusively, the kinetic parameters assessed in our study included the following sequential steps: time required for the plasma membrane to be permeabilized, time to cross the permeabilized plasma membrane, time to cross cytosol, time to cross nuclear pores, and finally the time for the intercalating dyes to bind DNA and equilibrate. Consequently, the measured kinetic parameters result in a sequence of events instead of only relating to plasma membrane transport coefficients. Nevertheless, there is evidence that the characteristic times of cytosol diffusion [29] and nuclear membrane crossing [30] are far shorter than these of plasma membrane crossing. Hence, SYTOX Green uptake is limited primarily by the permeability of the plasma membrane. Therefore, it can be argued that the rate constants obtained in our study to a good approximation describe transportation across the plasma membrane for this model drug system. 
The observation of single nuclei and their individual signal intensity enhancement could be of interest for a statistical approach. Different factors could influence the standard deviations of the kinetic parameters in the cell population, especially the spatial distribution of microbubbles on the microscopic scale, and their proximity to each of the cells. To assess model drug uptake in relation to the microbubble density, a cell membrane staining could be performed with DiD fluorescent dye which allows measuring the fraction of model drug-positive cells. However, this was outside the scope of the current study because the FCFM system was only capable of acquiring in a single spectral band. In addition, a previous study [17] showed that microbubbles need to be in close contact with plasma membranes in order for the cavitation effect to induce a plasma membrane permeabilization. This vicinity-mediated permeabilization probably influences the nature of the uptake, entailing heterogeneity in the resulting rate constants. Besides, evaluation of cell viability will be the subject of further studies in order to investigate the effects of US pressure on the cells [31,32].

\section{Conclusion}

In conclusion, the feasibility of real-time monitoring of USmediated intracellular delivery and its quantitative assessment has been demonstrated with FCFM for the first time. An in vitro setup was designed to allow a continuous monitoring of US-mediated drug model uptake before, during, and after US exposure. This study opens road to further investigation, especially the influence of acoustic parameters on the kinetic parameters and the translation to in vivo experiments. FCFM has great potential for in vivo minimally invasive applications in the context of local drug delivery.

Acknowledgments. We are grateful to Dr. J.R. Cazalets and colleagues (INCIA UMR 5287/University Bordeaux 2, France) for letting us use their cell culture facility. This study was supported by EU project SonoDrugs (FP7-NMP4-LA-2008-213706), ERC project 268906 "Sound Pharma", and Foundation InNaBioSanté- project ULTRAFITT.

Conflict of interest. The authors declare that they have no conflict of interest.

\section{References}

1. Allen TM, Cullis PR (2004) Drug delivery systems: entering the mainstream. Science 303:1818-1822

2. Yeh ETH (2004) Cardiovascular complications of cancer therapy: diagnosis, pathogenesis, and management. Circulation 109:3122-3131

3. Krishna R, Yu L (2008) Biopharmaceutics applications in drug development. Springer, New York

4. Skauen DM, Zentner GM (1984) Phonophoresis. Int J Pharm 20:235-245

5. Miller MW, Miller DL, Brayman AA (1996) A review of in vitro bioeffects of inertial ultrasonic cavitation from a mechanistic perspective. Ultrasound Med Biol 22:1131-1154

6. Mitragotri S (2005) Healing sound: the use of ultrasound in drug delivery and other therapeutic applications. Nat Rev Drug Discov 4:255-260

7. Sundaram J, Mellein BR, Mitragotri S (2003) An experimental and theoretical analysis of ultrasound-induced permeabilization of cell membranes. Biophys J 84:3087-3101
8. Ter Haar G (2007) Therapeutic applications of ultrasound. Prog Biophys Mol Biol 93:111-129

9. Krasovitski B, Frenkel V, Shoham S et al (2011) Intramembrane cavitation as a unifying mechanism for ultrasound-induced bioeffects. PNAS 108(8):3258-3263

10. Ogawa K, Tachibana K, Uchida $T$ et al (2001) High-resolution scanning electron microscopic evaluation of cell-membrane porosity by ultrasound. Med Electron Microscopy 34:249-253

11. Mehier-Humbert S, Bettinger T, Yan F et al (2005) Plasma membrane poration induced by ultrasound exposure: implication for drug delivery. J Control Release 104(1):213-222

12. White NS, Errington RJ (2005) Fluorescence techniques for drug delivery research: theory and practice. Adv Drug Deliv Rev 57:17-42

13. Staruch R, Chopra R, Hynynen K (2011) Localised drug release using MRI-controlled focused ultrasound hyperthermia. Int J Hyperthermia 27 (2):156-171

14. Dreher MR, Liu W, Michelich CR et al (2006) Tumor vascular permeability, accumulation, and penetration of macromolecular drug carriers. J Natl Cancer Inst 98:335-344

15. Deckers R, Yudina A, Cardoit LC et al (2011) A fluorescent chromophore TOTO-3 as a "smart probe" for the assessment of ultrasound-mediated local drug delivery in vivo. Contrast Media Mol Imaging 6:267-274

16. Chin CT, Lancee C, Borsboom J et al (2003) Brandaris 128: a digital 25 million frames per second camera with 128 highly sensitive frames. Rev Sci Instrum 74:5026-5034

17. Van Wamel A, Kooiman K, Harteveld M et al (2006) Vibrating microbubbles poking individual cells: drug transfer into cells via sonoporation. J Control Release 112:149-155

18. Kooiman K, Foppen-Harteveld M, Van der Steen AFW et al (2011) Sonoporation of endothelial cells by vibrating targeted microbubbles. J Control Release 154:35-41

19. Al-Gubory KH, Houdebine LM (2006) In vivo imaging of green fluorescent protein-expressing cells in transgenic animals using fibred confocal fluorescence microscopy. Eur J Cell Biol 85:837-845

20. Luciani A, Wilhelm C, Bruneval P et al (2009) Magnetic targeting of iron-oxide-labeled fluorescent hepatoma cells to the liver. Eur Radiol 19:1087-1096

21. Lin KY, Maricevich M, Bardeesy N et al (2008) In vivo quantitative microvasculature phenotype imaging of healthy and malignant tissues using a fiber-optic confocal laser microprobe. Transl Oncol 1:84-94

22. Yudina A, Lepetit-Coiffé M, Moonen CTW (2010) Evaluation of the temporal window for drug delivery following ultrasoundmediated membrane permeability enhancement. Mol Imaging Biol 13:239-249

23. Lepetit-Coiffé M, Yudina A, Lourenço de Oliveira P et al (2009) Correlation of ultrasound-mediated drug delivery with acoustical properties of the transducer by macroscopic fluorescence imaging. ISTUarticlet, Aix-en-Provence, France

24. Greis C (2004) Technology overview: SonoVue (Bracco, Milan). Eur Radiol 14:11-15

25. Sung KB, Richards-Kortum R, Follen M et al (2003) Fiber optic confocal reflectance microscopy: a new real-time technique to view nuclear morphology in cervical squamous epithelium in vivo. Opt Express 11:3171-3181

26. Hallow DM, Mahajan AD, Prausnitz MR (2007) Ultrasonically targeted delivery into endothelial and smooth muscle cells in ex vivo arteries. J Control Release 118:285-293

27. Papenfuss HD, Gross JF, Intaglietta M et al (1979) A transparent access chamber for the rat dorsal skin fold. Microvasc Res 18:311-318

28. Lehr HA, Leunig M, Menger MD et al (1993) Dorsal skinfold chamber technique for intravital microscopy in nude mice. Am J Pathol 143:1055-1062

29. Horowitz SB (1972) The permeability of the amphibian oocyte nucleus, in situ. J Cell Biol 54:609-625

30. Gerace L, Burke B (1988) Functional organization of the nuclear envelope. Ann Rev Cell Biol 4:335-374

31. Huber PE, Pfisterer P (2000) In vitro and in vivo transfection of plasmid DNA in the Dunning prostate tumor R3327-AT1 is enhanced by focused ultrasound. Gene Ther 7:1516-1525

32. Yudina A, de Smet M, Lepetit-Coiffé M et al (2011) Ultrasoundmediated intracellular drug delivery using microbubbles and temperature-sensitive liposomes. J Control Release 155:442-448 